\title{
Experimental Comparison of Observers for Tool Position Estimation of Industrial Robots
}

\author{
Robert Henriksson, Mikael Norrlöf, Stig Moberg, Erik Wernholt, Thomas B. Schön
}

\begin{abstract}
This paper investigates methods for tool position estimation of industrial robots. It is assumed that the motor angular position and the tool acceleration are measured. The considered observers are different versions of the extended Kalman filter as well as a deterministic observer. A method for tuning the observers is suggested and the robustness of the methods is investigated. The observers are evaluated experimentally on a commercial industrial robot.
\end{abstract}

\section{INTRODUCTION}

Current industrial robot development is focused on increasing the robot performance, reducing the robot cost, improving safety, and introducing new functionalities as described in [1]. The need for cost reduction results in the use of cost optimized robot components with increased elasticity and larger individual variation, such as variation of gearbox stiffness or in the parameters describing the mechanical arm. Cost reduction also implies weight-optimized robots and thus lower mechanical stiffness and more complicated vibration modes. To maintain or improve the robot performance, the motion control must be improved for this new generation of robots. For robots with traditional measurement systems, where only the motor angular position is measured, this can be obtained by improving the model-based control as described in [2]. Another option is to use inertial sensors to improve the estimation of the robot tool position. This paper investigates how the tool position can be estimated by the use of observers.

One early contribution is [3], which describes how the nonlinear dynamics of elastic robots can be handled. The problems of gravity compensation for elastic robots is studied in [4]. One commonly used observer is the linear Kalman filter $(\mathrm{KF})$ or the extended Kalman filter $(\mathrm{EKF})$, used for nonlinear systems. A KF is used for a single-axis robot arm in [5] and [6]. EKFs are used in [7], and also in [8], where a two-axis robot, with tool position and joint speed measurements, are used. Estimation using motor measurements only is studied in [9]. In [10], accelerometers are used, and estimation is performed by particle filters as well as EKFs. A deterministic observer, utilizing accelerometers, is described in [11].

The estimated tool position can be used for on-line feedback control as a mean of increasing both the robust and the nominal performance of the robot. Another possible use

This work was supported by ABB AB - Robotics, Vinnova's Industry Excellence Center LINK-SIC at Linköping University, and the Swedish Research Council (VR), which is gratefully acknowledged.

All authors are with the Department of Electrical Engineering, Linköping University, SE-58183 Linköping, Sweden \{mino, stig, erikw, schon\} disy.liu.se. of tool estimation is iterative learning control (ILC) [12]. In [13] it is shown that motor side learning is insufficient if the mechanical resonances are exited by the robot trajectory. Other applications in need of tool position estimation are, e.g., model identification, supervision, diagnostics, and automatic controller tuning.

The estimation problem can be divided into one static (low frequencies) and one dynamic (mid to high frequencies) estimation problem. A large industrial robot typically has a static volumetric accuracy of $2-15 \mathrm{~mm}$ due to gravity deflection, component tolerance, and variations in the assembly procedure. One common solution to the static problem is to perform an off-line identification of an extended kinematic model and an elasto-static model, i.e., to solve the problem by model-based control. In this way a static accuracy of $0.5 \mathrm{~mm}$ can be obtained. Thus, tool estimation by observers is most interesting to investigate for the dynamic problem, i.e., for frequencies larger than, e.g., $1 \mathrm{~Hz}^{1}$. The static and dynamic estimates can then be fused, for example, in the frequency domain.

This paper investigates methods for estimation of the robot tool position. It is assumed that the motor angular position and the tool acceleration are measured. Primarily, the dynamic estimation problem is treated but the static estimation problem is also briefly discussed. The observers considered are different versions of the EKF as well as the deterministic observer described in [11]. A method for tuning the observers is suggested and the robustness of the methods is investigated. The observers are evaluated experimentally by using two axes of an industrial robot. However, the methods can easily be adapted to a six degrees-of-freedom (DOF) industrial robot.

\section{Flexible Robot Model}

The overall idea in this work is to investigate the use of an accelerometer in order to improve the estimate of the robot tool position. The estimators that will be introduced in the subsequent section all rely on an accurate model of the robot. Before we give the state-space model in Section II-B, the relevant coordinate frames are introduced in Section II-A.

\section{A. Coordinate Frames}

The robot geometry is described using the world $(w)$ coordinate frame, which is fixed in the robot base. The absolute position of the robot tool $p^{w}$ is resolved in this frame. The accelerometer is attached to the robot tool, implying that it

\footnotetext{
${ }^{1}$ The frequency should be well below the lowest mechanical resonance of the robot.
} 


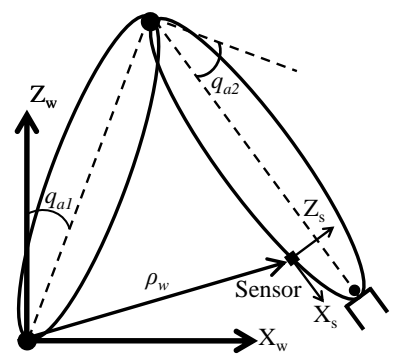

Fig. 1. Coordinate frames used in modeling the 2-DOF robot. The world $(w)$ frame is attached to the robot base and the sensor $(s)$ frame is attached to the accelerometer.

is moving with the robot. The sensor coordinate frame $(s)$ is attached to the accelerometer. For an illustration of the involved coordinate frames we refer to Fig. 1. The two arm angles $q_{\mathrm{a} 1}$ and $q_{\mathrm{a} 2}$ are defined relative to the fixed reference provided by the world frame.

\section{B. State-Space Model}

A robot is always subject to a load due to its own weight and possibly also due to exterior forces. This implies that the compliance of the structure will result in a certain deflection. This compliance is also referred to as elasticity and it will in this work be modeled using flexible joints, whereas the links are still considered being rigid. In order to accomplish this it is no longer sufficient to use only one angle per joint, as discussed above. Instead each joint will be described using two angles, the arm angle $q_{\mathrm{a} i}$, previously introduced, and the motor angle $q_{\mathrm{m} i}$. Now, the difference $q_{\mathrm{a} i}-q_{\mathrm{m} i}$ describes the deflection in joint $i$.

The state vector is given by

$$
x=\left(\begin{array}{llll}
q_{\mathrm{a}}^{T} & q_{\mathrm{m}}^{T} & \dot{q}_{\mathrm{a}}^{T} & \dot{q}_{\mathrm{m}}^{T}
\end{array}\right)^{T},
$$

where $q_{a}=\left(\begin{array}{ll}q_{\mathrm{a} 1} & q_{\mathrm{a} 2}\end{array}\right)^{T}, q_{m}=\left(\begin{array}{ll}q_{\mathrm{m} 1} & q_{\mathrm{m} 2}\end{array}\right)^{T}$, contain the arm angles $q_{\mathrm{a}}$ and the motor angles $q_{\mathrm{m}}$ of both joints. In order to get a feeling for the model, we provide it in state-space form below. Engaging in a full description and derivation of the model falls outside the scope of this paper. However, the interested reader can refer to [14] for all the details. The model accounts for flexibilities in the joints via nonlinear stiffness, nonlinear friction and linear viscous damping.

$$
\dot{x}=\left(\begin{array}{c}
x_{3} \\
x_{4} \\
M_{a}^{-1}\left(x_{1}\right)\left(-C\left(x_{1}, x_{3}\right)-G\left(x_{1}\right)-A(x)\right) \\
M_{m}^{-1}\left(A(x)+\kappa\left(x_{4}\right)+u\right)
\end{array}\right)
$$

where $A(x)=D\left(x_{3}-x_{4}\right)+\tau_{s}\left(x_{1}, x_{2}\right) . A(x)$ accounts for the flexibilities in the joints, via the linear viscous damping $D\left(x_{3}-x_{4}\right)$ and the nonlinear stiffness $\tau_{s}\left(x_{1}, x_{2}\right)$. In other words, if we dispense with $A(x)$, we are back at a standard rigid robot model. Furthermore, $M_{a}\left(x_{1}\right)$ and $M_{m}$ are the mass matrices for the arm and motor, $C\left(x_{1}, x_{3}\right)$ accounts for the centrifugal and centripetal torques, and $G\left(x_{1}\right)$ accounts for the effect of gravity on the links. The nonlinear friction is described by $\kappa\left(x_{3}\right)$ and $u$ represents the motor torque applied to the robot. All the estimators introduced in this paper are based on this model or a simplified version of it, as is further described in the subsequent section.

\section{Estimation}

Given the general nonlinear model (2) the estimation of the unknown states can be made in many different ways. Here two main approaches are investigated. The first is to use an extended Kalman filter [15] and this results in three different implementations, where the model and the measurements are used differently. The second approach is to use a nonlinear deterministic observer [11], where the model structure is explicitly used to create an observer based upon motor position and accelerometer measurements.

\section{A. Extended Kalman Filter}

The EKF addresses the estimation problem for a general nonlinear discrete-time system,

$$
\begin{aligned}
x_{k+1} & =F\left(x_{k}, u_{k}\right)+v_{k}, & & v_{k} \sim \mathcal{N}\left(0, Q_{k}\right), \\
z_{k} & =h\left(x_{k}, u_{k}\right)+w_{k}, & & w_{k} \sim \mathcal{N}\left(0, R_{k}\right) .
\end{aligned}
$$

In order to compute estimates of the states, the system is linearized around the previous estimate and the EKF is implemented as a two-step procedure, consisting of measurement update

$$
\begin{aligned}
& \hat{x}_{k \mid k}=\hat{x}_{k \mid k-1}+K_{k}\left(z_{k}-h\left(\hat{x}_{k \mid k-1}, u_{k}\right)\right), \\
& P_{k \mid k}=P_{k \mid k-1}-K_{k} H_{k} P_{k \mid k-1},
\end{aligned}
$$

and the time update

$$
\begin{aligned}
\hat{x}_{k+1 \mid k} & =F\left(\hat{x}_{k \mid k}, u_{k}\right), \\
P_{k+1 \mid k} & =A_{k} P_{k \mid k} A_{k}^{T}+Q_{k}, \\
K_{k} & =P_{k \mid k-1} H_{k}^{T}\left(H_{k} P_{k \mid k-1} H_{k}^{T}+R_{k}\right)^{-1} .
\end{aligned}
$$

The following representation is used for the linearized system and output matrices,

$$
A_{k}=\left.\frac{\partial F\left(x, u_{k}\right)}{\partial x}\right|_{x=\hat{x}_{k \mid k}}, \quad H_{k}=\left.\frac{\partial h\left(x, u_{k}\right)}{\partial x}\right|_{x=\hat{x}_{k \mid k-1}} .
$$

In Section III-C a method to find values of the noise covariances $Q_{k}$ and $R_{k}$ from data will be outlined. Three different implementations of the EKF are used throughout the rest of the paper. The nonlinear continuous-time model is discretized using an Euler forward difference approximation.

1) EKF Complete: In the EKF Complete the model described in Section II is fully utilized, including friction and nonlinear stiffness. The measurement equation (3b) includes the motor measurements as well as the accelerometer measurements,

$$
h\left(x_{k}, u_{k}\right)=\left[\begin{array}{c}
x_{2 k} \\
\ddot{\rho}_{s}\left(x_{k}\right)
\end{array}\right],
$$

where

$$
\ddot{\rho}_{s}\left(x_{k}\right)=R_{w}^{s}\left(x_{1 k}\right)\left(J\left(x_{1 k}\right) \dot{x}_{3 k}+\dot{J}\left(x_{1 k}\right) x_{3 k}+G_{w}\right) .
$$

Since $\dot{x}_{3}$ is not a state, it is replaced by the $\dot{x}_{3}$ equation in (2). $J\left(x_{1}\right)$ is the Jacobian of the manipulator kinematics and $G_{w}$ is the gravity vector measured by the accelerometer. 
2) EKF Motor: The EKF Motor is used as a base line for what can be achieved without having the additional accelerometer sensor. The measurement equation uses only the motor angular positions,

$$
h\left(x_{k}, u_{k}\right)=x_{2 k}
$$

while the dynamic model is fully utilized.

3) EKF Integrated: What characterizes the EKF Complete and the corresponding measurement equation (6), is that the dynamics appears explicitly in the expression for the accelerometer measurement. In the EKF Integrated the accelerometer measurement is rotated into the fixed frame and then integrated to get a velocity,

$$
\dot{\rho}_{w}^{M}(k)=\int_{0}^{t_{k}} \ddot{\rho}_{w}^{M}(t) d t=\int_{0}^{t_{k}} R_{s}^{w}(t) \ddot{\rho}_{s}^{M}(t)-G_{w} d t .
$$

The integral is solved numerically from the discrete-time data. The measurement equation now becomes,

$$
h\left(x_{k}, u_{k}\right)=\left[\begin{array}{c}
x_{2 k} \\
J\left(x_{1 k}\right) x_{3 k}
\end{array}\right],
$$

which is much simpler than the one in (6). The explicit dependence on the dynamic model is now removed from the measurement equation. Instead it only depends on the kinematic model which, in general, has much less uncertainty.

\section{B. Deterministic Observer}

The EKFs are compared with the result from a deterministic observer by de Luca et al. [11]. A very important characteristic of the deterministic observer is the computational complexity, which is significantly lower than the EKF. This implies that the deterministic observer could be used in feedback implementations while the EKF, with the available computer power in current commercial industrial manipulators, only can be aimed at off-line computations for controller tuning or ILC. The deterministic observer in [11] relies heavily on the model structure and it is assumed that the robot is an $N$-DOF robot with linear joint elasticity. The system equation (2) can be transformed into

$$
\dot{x}=\left[\begin{array}{c}
x_{3} \\
x_{4} \\
0 \\
M_{m}^{-1}\left(K\left(x_{1}-x_{2}\right)+D\left(x_{3}-x_{4}\right)\right)
\end{array}\right]+\left[\begin{array}{c}
0 \\
0 \\
f_{\text {nonlin }}(x) \\
M_{m}^{-1} u
\end{array}\right]
$$

where $K\left(x_{1}-x_{2}\right)$ is a linear approximation of $\tau_{s}\left(x_{1}, x_{2}\right)$,

$$
\begin{aligned}
f_{\text {nonlin }}(x)= & M_{a}^{-1}\left(x_{1}\right)\left(-C\left(x_{1}, x_{3}\right)-G\left(x_{1}\right)\right. \\
& \left.-K\left(x_{1}-x_{2}\right)-D\left(x_{3}-x_{4}\right)\right),
\end{aligned}
$$

and the state is defined according to (1). Now use the equation for the acceleration measurement in (7), which can be solved for $\dot{x}_{3}$ when $R_{w}^{s}\left(x_{1}\right) J\left(x_{1}\right)$ is non-singular. Let $\ddot{q}_{a}^{M}$ be the computed acceleration value,

$$
\ddot{q}_{a}^{M}=\left(R_{w}^{s}\left(x_{1}\right) J\left(x_{1}\right)\right)^{-1}\left(\ddot{\rho}_{s}^{M}-R_{w}^{s}\left(x_{1}\right)\left(\dot{J}\left(x_{1}\right) x_{3}+G_{w}\right)\right) .
$$

An observer for the system can then be expressed as

$$
\dot{\hat{x}}=A_{0} \hat{x}+G_{0}\left[\begin{array}{c}
\ddot{q}_{a}^{M}\left(\ddot{\rho}_{s}^{M}, \hat{x}\right) \\
u
\end{array}\right]+L\left(q_{m}^{M}-C_{0} \hat{x}\right),
$$

where $L \in \mathbb{R}^{4 N \times N}$ and $C_{0} \in \mathbb{R}^{N \times 4 N}$ are defined to get a well behaved observer, and so that $C_{0} \hat{x}=\hat{q}_{m}$. The choice of poles for the deterministic observer is discussed in the next section.

\section{Automatic Tuning}

1) EKF: The tuning of the noise covariances in the different EKF implementations can be stated as a general system identification problem. It is here solved by minimizing the prediction error using a set of measurement data where the motor angles and the tool acceleration are available, together with measurements of the true tool position. The covariance matrices are parameterized as

$$
\begin{aligned}
\widetilde{R}_{\lambda} & =\left[\begin{array}{cccc}
\lambda_{1} I^{2 \times 2} & 0 & 0 & 0 \\
0 & \lambda_{2} I^{2 \times 2} & 0 & 0 \\
0 & 0 & \lambda_{3} I^{2 \times 2} & 0 \\
0 & 0 & 0 & \lambda_{4} I^{2 \times 2}
\end{array}\right] \widetilde{R}, \\
\widetilde{W}_{\lambda} & =\left[\begin{array}{cc}
\lambda_{5} I^{2 \times 2} & 0 \\
0 & I^{2 \times 2}
\end{array}\right] \widetilde{W},
\end{aligned}
$$

where $\widetilde{R}$ and $\widetilde{W}$ represent initial guesses and $\lambda_{i}, i=$ $1, \ldots, 5$ are free variables in the optimization. The objective function is to minimize the 2-norm of the prediction error in the two Cartesian dimensions. The resulting optimization problem is solved using the COMPLEXRF algorithm [16], which is a heuristic optimization method. It has a buildin randomization component that should help to avoid local minima. However, there is no guarantee for the algorithm to result in a global minimum.

2) Deterministic Observer: For the deterministic observer eight poles have to be specified and to solve this an optimization problem is posed and solved. The objective function and the optimization algorithm, COMPLEXRF [16], are the same as in the EKF tuning. However, the constraints and the free variables are different, and the poles are parameterized in terms of their eigenfrequency $\omega_{i}>0$ and damping $\zeta_{i}>0$. No guarantee for the optimization algorithm to converge to a global optimum exists. However, in the next section it will be clear that a sufficiently good observer can be found with this technique.

\section{EXPERIMENTAL RESULTS}

\section{A. Experimental Setup}

The experimental comparison of the observers will be carried out by using measurements collected from a 6-DOF $\mathrm{ABB}$ industrial robot where only the second and third joints are used for the motions, see Fig. 2. An analog 3-axis Crossbow accelerometer from the GP Series [17] is attached to the robot tool and integrated with the standard robot control system. The robot tool is rigid and has maximum allowed weight for the robot. Measurement data from the resulting motor torques, motor angular positions, and tool acceleration are sampled at $2 \mathrm{kHz}$ and exported to MATLAB. To evaluate the performance of the observers, the tool position is also measured by a Leica laser tracker [18], which gives measurement data at $1 \mathrm{kHz}$ with an accuracy of $0.01 \mathrm{~mm}$. 

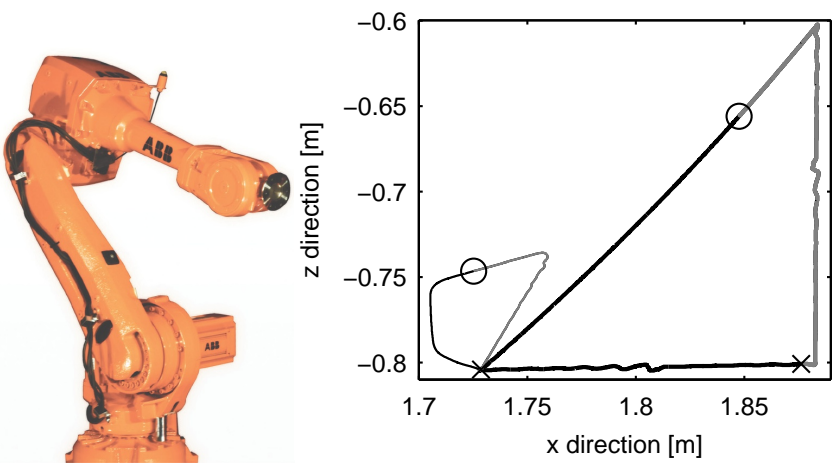

Fig. 2. Left: The ABB 6-DOF manipulator IRB4600 used in the experiments. Right: Illustration of the two different paths and their location in the robot workspace. Thick line: P1, thin line: P2. Gray line: selected part showed in Fig. 4-6, where 'o' indicates the start and ' $x$ ' the end of the selected part.

For the evaluation, the following two data sets will be used, corresponding to different paths of the robot tool:

P1: A triangular path according to Fig. 2 with a motion of $15-20 \mathrm{~cm}$

P2: A path according to Fig. 2, which is a smaller motion compared to $\mathrm{P} 1$, and located in a slightly different part of the working area to evaluate robustness.

To create external disturbance forces, the tool is also hit a couple of times by a rubber hammer in both paths. Note that the model parameters that are used by the observers in the experiments come from a separate identification procedure (see, e.g., [19]). This means that they are not tuned to obtain optimal performance for the observers. It is only the noise model that is tuned for the EKFs (and observer poles for the deterministic observer) according to Section III-C. This is in contrast to [11], where the model parameters are included in the tuning of the deterministic observer. This could also be carried out here, both for the EKFs and the deterministic observer, and the resulting solution would then be closely related to identification using nonlinear predictionerror methods [20].

\section{B. Nominal Performance}

In this section, the four different observers from Section III will be evaluated on path $\mathrm{P} 1$. The covariance matrices for each of the three EKFs, as well as the poles of the deterministic observer, are optimized according to Section III$\mathrm{C}$ by using the measurement data. This is done in order to get comparable results for the different observers. As comparison, the tool position is also calculated from the motor angles by using the rigid robot kinematics, ignoring all static and dynamic elastic effects.

As can be seen in Fig. 3, it is obvious that the estimation of the tool position is insufficient. The performance of all the observers is worse than if using the rigid robot kinematics, and especially EKF Integrated and the deterministic observer have a major drift. However, it is important to note that this result was expected for several reasons. Firstly, to get

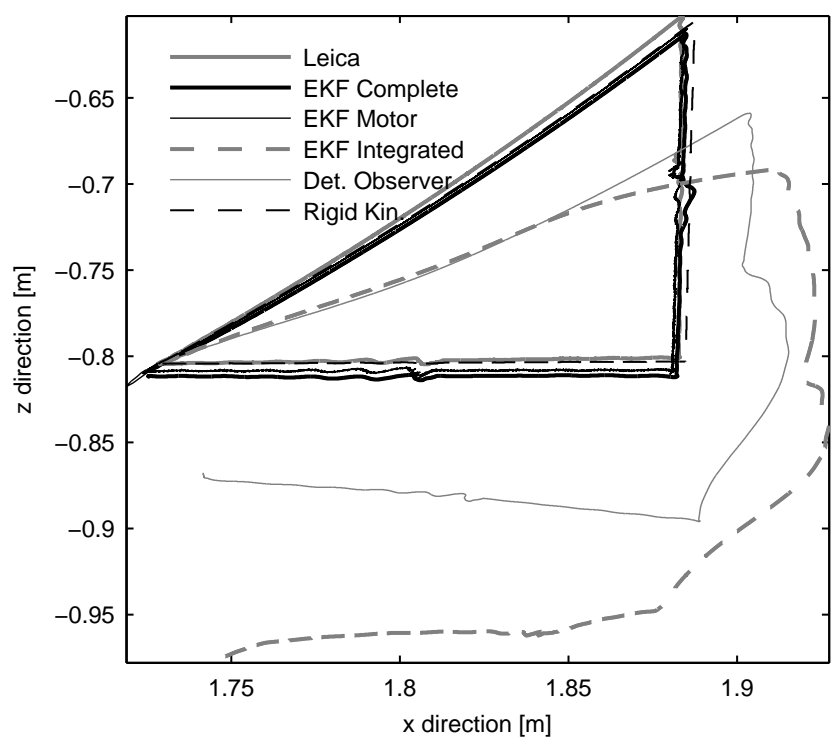

Fig. 3. Estimated tool position for path P1. The four observers are plotted together with the true position (measured by the Leica laser tracker) and the estimate using rigid robot kinematics.

a good estimate of the static or low-frequency behavior the model must be very accurate, including a calibrated elastostatic kinematic model that describes kinematic errors as well as deflections due to gravitational forces. Secondly, the observers are tuned for mid-range frequencies, in this case $3-30 \mathrm{~Hz}$, since that is an important frequency range where the current techniques (e.g. rigid robot kinematics) fail to produce sufficiently accurate estimates. To emphasize the mid-range frequencies the estimated tool position is therefore filtered with a bandpass filter. The result can be seen in Fig. 4 and Table I, where the max and mean error are given for all the observers. EKF Integrated is omitted in the bandpassfiltered figures, but gives almost indistinguishable results as the deterministic observer (see Table I). From Table I it is evident that the observers give superior estimates for midrange frequencies, compared to only using the rigid robot kinematics (a factor 2-4 in reduced mean error). In terms of $\max$ error, EKF Motor is best, mainly due to the response of the hammer strike at time 1.5-1.7 s in Fig. 4. However, in terms of mean error and at other time instances, EKF Motor gives a noisy and less accurate estimate compared to the other observers. EKF Integrated and the deterministic observer give the best mean error, followed by EKF Complete. To see the differences between the observers more clearly, we will next consider how sensitive the performance is to the observer tuning and model errors.

\section{Robustness}

Since the observers in Section IV-B were tuned for that particular path before the actual estimation, the immediate question is what happens if they are used under slightly different conditions. Therefore the same tuning as in Section IV-B is used, but the observers are applied to path P2. 
TABLE I

MAX AND MEAN ESTIMATION ERROR (IN MILLIMETERS) FOR THE DIFFERENT OBSERVERS, BOTH FOR ALL FREQUENCIES AND THE MID-RANGE FREQUencies 3-30 Hz. All observers aRe tuned For PATH P1 AND EVAluated on PATH P1 (Nominal), PATH P2 (Tuning), and PATH P1 With REDUCED STIFFNESS PARAMETERS (Model Errors).

\begin{tabular}{|l|r|r|r|r|r|r|r|r|r|r|r|}
\hline \multicolumn{2}{|c|}{} & \multicolumn{2}{|c|}{ EKF Complete } & \multicolumn{2}{|c|}{ EKF Motor } & \multicolumn{2}{|c|}{ EKF Integrated } & \multicolumn{2}{|c|}{ Det. Observer } & \multicolumn{2}{|c|}{ Rigid kinematics } \\
\cline { 3 - 12 } \multicolumn{2}{|c|}{} & Max & Mean & Max & Mean & Max & Mean & Max & Mean & Max & Mean \\
\hline \multirow{2}{*}{ Nominal } & All & 17.6 & 9.7 & 16.2 & 7.2 & 174.3 & 101.0 & 97.0 & 59.9 & 5.6 & 2.7 \\
\cline { 2 - 13 } & $3-30 \mathrm{~Hz}$ & 1.007 & 0.070 & 0.914 & 0.111 & 1.114 & 0.056 & 1.121 & 0.057 & 2.142 & 0.221 \\
\hline \multirow{2}{*}{ Tuning } & All & 9.4 & 7.2 & 16.3 & 6.0 & 82.0 & 36.4 & 43.3 & 28.6 & 2.1 & 0.5 \\
\cline { 2 - 12 } & $3-30 \mathrm{~Hz}$ & 0.135 & 0.027 & 0.698 & 0.122 & 0.110 & 0.018 & 0.108 & 0.020 & 1.351 & 0.219 \\
\hline $\begin{array}{l}\text { Model } \\
\text { Errors }\end{array}$ & All & 24.0 & 16.3 & 24.0 & 13.2 & 163.3 & 94.3 & 69.8 & 45.4 & 5.6 & 2.7 \\
\cline { 2 - 11 } & $3-30 \mathrm{~Hz}$ & 1.070 & 0.090 & 2.803 & 0.340 & 1.113 & 0.056 & 1.120 & 0.057 & 2.142 & 0.221 \\
\hline
\end{tabular}

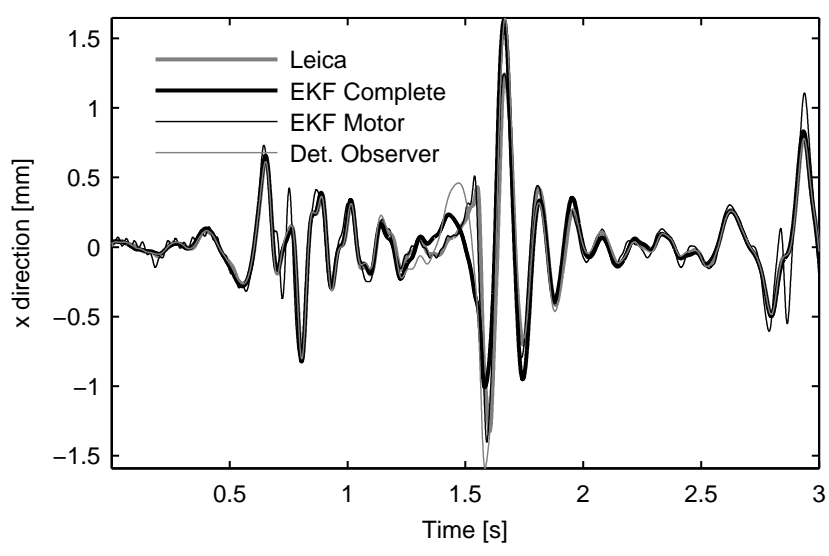

Fig. 4. Estimated tool $x$-position, band-pass filtered to $3-30 \mathrm{~Hz}$, for a selected part of path P1. The results from the observers are plotted together with the true position (measured by the Leica laser tracker).

The estimated tool position is still poor in terms of static accuracy, with a similar behavior as in Fig. 3. The bandpass filtered result can be seen in Fig. 5. From Table I, the major conclusion is that EKF Motor is really sensitive to the tuning. In the nominal case, the mean error is increased by $2 \%$ (deterministic observer), 25\% (EKF Complete), and 98\% (EKF Motor) compared to the EKF Integrated. For path P2, these numbers are $11 \%, 50 \%$, and $580 \%$.

Another important practical question is how robust the observers are to model errors. The joint stiffness parameters are therefore reduced by $50 \%$ and the result can be seen in Fig. 6 and Table I. Now the differences between the observers are even more obvious. For mid-range frequencies, EKF Integrated and the deterministic observer are not affected at all in terms of mean and max error. For EKF Motor and EKF Complete the mean error is increased with $206 \%$ and $29 \%$, respectively, compared to the nominal case. The advantage of integrating the accelerometer signal is therefore evident. Since only the robot kinematic model is used in the measurement equation (8) for EKF Integrated, the errors are much smaller compared to the other EKFs that rely more heavily on a correct description of the dynamics. EKF Motor gives extremely poor estimates, as could be expected since it relies entirely on the motor measurements and the

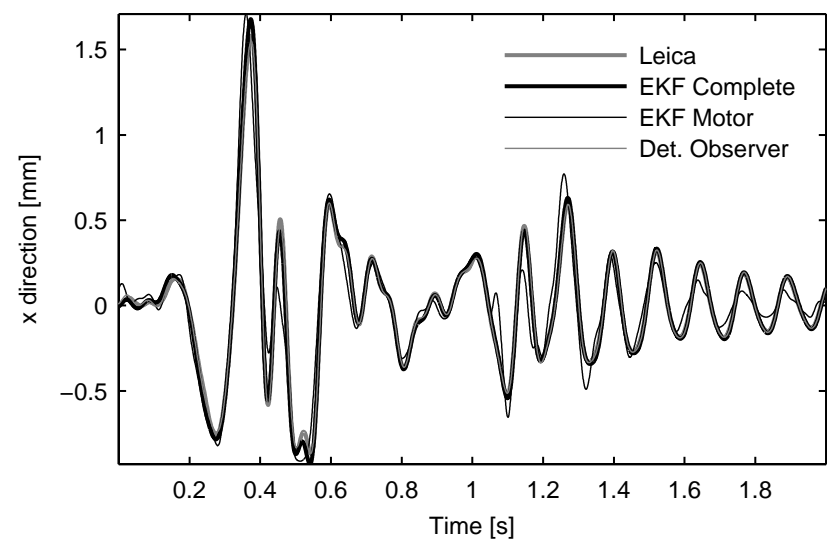

Fig. 5. Estimated tool $x$-position, band-pass filtered to $3-30 \mathrm{~Hz}$, for a selected part of path $\mathrm{P} 2$. The observers are plotted together with the true position (measured by the Leica laser tracker).

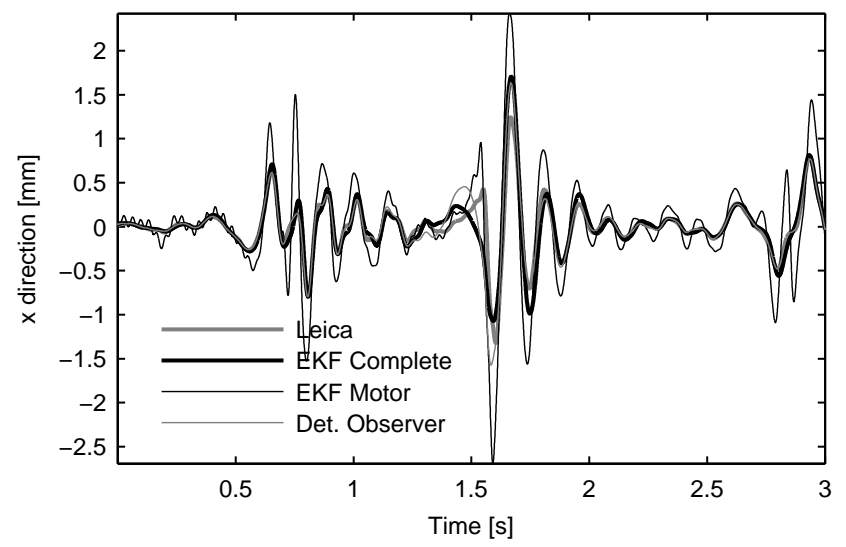

Fig. 6. Estimated tool $x$-position, band-pass filtered to $3-30 \mathrm{~Hz}$, for a selected part of path P1. The observers have a stiffness error of $50 \%$ and are plotted together with the true position (measured by the Leica laser tracker). 
dynamic model to estimate the tool position. EKF Complete slightly distorts the accelerometer measurements through its complicated measurement equation (6) that depends on the dynamics.

\section{Discussion}

The computational complexity varies quite much between the different observers. This, of course, depends on the implementation, but to get a flavor, EKF Complete requires $4.9 \mathrm{~s}$ to estimate $1 \mathrm{~s}$ of data, EKF Integrated $2.6 \mathrm{~s}$, EKF Motor $2.1 \mathrm{~s}$, and the deterministic observer $0.17 \mathrm{~s}$. Increasing the model complexity to 3 or 6 DOF will of course demand much more computational resources and the deterministic observer is therefore really interesting if real-time implementation becomes an issue.

The stability problem (drift) of the deterministic observer and EKF Integrated must be solved to make them practically useful. For the deterministic observer, the singularity problem associated with the inverse Jacobian must also be handled.

\section{COnClusions And Future Work}

The main conclusion from this experimental comparison is that accelerometers in combination with observers can significantly increase the accuracy of the tool position estimate compared to simply using the motor position and the kinematics, or only using the motor position measurement and an observer. Except in the nominal case none of the observers produced very good estimation results at low frequencies and the results showed a large drift when introduced to model errors. The best results for low frequencies were achieved by EKF Complete and EKF Motor, but since the tuning was performed to achieve a good result for mid-range frequencies, the result could be improved with a different tuning principle. With a calibrated kinematic model, the lowfrequency errors would also be drastically reduced. In the mid-frequency range that can be considered most important from a control perspective, for example for disturbance rejection and compensation of dynamic deflections, the observers performed very well. The best result was achieved by EKF Integrated, utilizing the complete dynamic model but with a simplified measurement equation where the accelerometer signal is integrated to get a speed signal. The deterministic observer also had a similar performance, but has proved to be slightly more sensitive to tuning errors. In some cases the deterministic observer even became unstable, which must be further investigated. The main advantage of the deterministic observer is the computational complexity which is at least an order-of-magnitude lower than for the EKFs.

A next step would be to fully use the 6-DOF capabilities of the robot and extend the robot model to this case. The computational complexity of the EKF will increase even more and therefore other implementations based on numerical computation of the Jacobian should be tested. One such example is the Unscented Kalman Filter [21]. The sensor system could be extended as well and a first step would be to include a gyro to get a 6-DOF measurement. Another important issue is the robustness with respect to trajectory and configuration in work area, i.e., to investigate the need for observer gain scheduling. Robustness for different robot individuals and tools must also be further studied. Another obvious future work is to calibrate the kinematic model to obtain accurate estimates also for low frequencies. This also includes to handle the drift in EKF Integrated and the deterministic observer.

\section{REFERENCES}

[1] T. Brogårdh, "Present and future robot control development-an industrial perspective," Annual Reviews in Control, vol. 31, no. 1, pp. $69-79,2007$.

[2] M. Björkman, T. Brogårdh, S. Hanssen, S.-E. Lindström, S. Moberg, and M. Norrlöf, "A new concept for motion control of industrial robots," in Proceedings of 17th IFAC World Congress, 2008, Seoul, Korea, July 2008

[3] S. Nicosia, P. Tomei, and A. Tornambe, "A nonlinear observer for elastic robots," IEEE Transactions on Robotics and Automation, vol. 4 , no. 1, pp. 45-52, Feb. 1988.

[4] A. De Luca and S. Panzieri, "An iterative scheme for learning gravity compensation in flexible robot arms," Automatica, vol. 30, no. 6, pp 993-1002, 1994.

[5] L. Alder and S. Rock, "Experiments in control of a flexible-link robotic manipulator with unknown payload dynamics: An adaptive approach," The International Journal of Robotics Research, vol. 13, no. 6, pp. 481-495, 1994.

[6] _ - "Frequency-weighted state estimation with application to estimation in the presence of sensor bias," IEEE Transactions on Control Systems Technology, vol. 4, no. 4, pp. 427-436, 1996.

[7] $\mathrm{Y}$. Li and $\mathrm{X}$. Chen, "End-point sensing and state observation of a flexible-link robot," IEEE/ASME Transactions on Mechatronics, vol. 6 , no. 3, pp. 351-356, 1994.

[8] V. Lertpiriyasuwat and M. Berg, "Extended kalman filtering applied to a two-axis robotic arm with flexible links," The International Journal of Robotics Research, vol. 19, no. 3, pp. 254-270, 2000.

[9] M. Jankovic, "Observer based control for elastic joint robots," IEEE Transactions on Robotics and Automation, vol. 11, no. 4, pp. 618-623, 1995.

[10] R. Karlsson and M. Norrlöf, "Position estimation and modeling of a flexible industrial robot," in Proceeding of the 16th IFAC World Congress, Prague, Czech Republic, July 2005.

[11] A. De Luca, D. Schröder, and M. Thümmel, "An accelerationbased state observer for robot manipulators with elastic joints," in Proceedings of 2007 IEEE International Conference on Robotics and Automation, Roma, Italy, Apr. 2007, pp. 3817-3823.

[12] J. Wallén, S. Gunnarsson, R. Henriksson, S. Moberg, and M. Norrlöf, "ILC applied to a flexible two-link robot model using sensor-fusionbased estimates," 2009, submitted to the 48th IEEE Conference on Decision and Control.

[13] J. Wallén, M. Norrlöf, and S. Gunnarsson, "Arm-side evaluation of ILC applied to a six-degrees-of-freedom industrial robot," in Proceedings of 17th IFAC World Congress 2008, Seoul, Korea, Seoul, Korea, July 2008, pp. $13450-13455$.

[14] S. Moberg, J. Öhr, and S. Gunnarsson, "A benchmark problem for robust control of a multivariable nonlinear flexible manipulator," in Proceedings of 17th IFAC World Congress, Seoul, Korea, July 2008.

[15] T. Kailath, A. Sayed, and B. Hassibi, Linear Estimation. Information and system science series. Prentice Hall,Upper Saddle River, New Jersey, 2000.

[16] M. Box, "A new method of constraint optimization and a comparison with other methods," The Computer Journal, vol. 8, pp. 42-52, 1965.

[17] Crossbow Technology, http://www.xbow.com, 2009.

[18] Leica Geosystems, http://www.leica-geosystems.com, 2009.

[19] E. Wernholt and S. Moberg, "Frequency-domain gray-box identification of industrial robots," in Proceedings of 17th IFAC World Congress, Seoul, Korea, July 2008, pp. 15372-15380.

[20] L. Ljung, System Identification: Theory for the User, 2nd ed. Upper Saddle River, New Jersey, USA: Prentice Hall, 1999.

[21] S. J. Julier and J. K. Uhlmann, "Unscented filtering and nonlinear estimation," Proceedings of the IEEE, vol. 92, no. 3, pp. 401-422, Mar. 2004. 